\title{
Study on Piecewise Linear Model of Anti-yaw Damper and Test Analysis
}

\author{
Dongxiao Yang ${ }^{1, a}$, Maoru Chi ${ }^{1, b}$, Wubin Cai ${ }^{1, c}$, Xun Wang ${ }^{1, d}$
}

${ }^{1}$ State Key Laboratory of Traction Power, Southwest Jiaotong University, Chengdu, China, 610031

ayangdx1990@163.com, ${ }^{\text {b }}$ cmr2000@163.com, cwubin0410@hotmail.com,
dwangxun0323@126.com

Keywords: railway vehicle; anti-yaw damper modelling; piecewise linear model; Maxwell model; dynamic characteristics

\begin{abstract}
In this paper a brief review is presented on the working principle of anti-yaw damper used widely on EMUs (Electric Multiple Units). Based on the Maxwell model, the piecewise linear model was developed by considering the rubber joint stiffness, the oil stiffness and the piecewise characteristics of hydraulic damper. Then, dynamic characteristic test for an anti-yaw damper was carried out, from which the dynamic damping curve and the dynamic stiffness curve has been achieved. At last, comparing with the test results and conventional Maxwell model, the piecewise linear model turned out to be able to accurately reflect the dynamic characteristics of the anti-yaw damper, whether unloading or not.
\end{abstract}

\section{Introduction}

The Anti-yaw damper is a kind of hydraulic shock absorber used widely on EMU vehicles, which can effectively inhibit hunting movement and play an important role in increasing the critical speed and improving the dynamic performance of the vehicle system. Its mathematical model is the basis of damper dynamic characteristics research and vehicle system dynamics simulation. Among them the equivalent parameter model is widely applied, such as the Maxwell model which consists of a liner stiffness ' $k$ ' in series with a linear damper ' $c$ ' [1]. Compared to the physical parameter model which need large number of structural parameters, fluid-solid coupling function, the Maxwell has advantages of more simplified and shorter calculation time on the dynamic simulation of high-speed trains [2-3].

However, the conventional Maxwell model of shock absorber, shown as Fig. 1(b), is not able to describe the dynamic characteristics accurately for ignoring the oil stiffness and piecewise damping characteristics. In this paper, firstly, a kind of Maxwell model is developed by considering the linear damping, rubber joint stiffness and oil stiffness. And the dynamic damping and dynamic stiffness is derived according to the relationship between the force and the displacement. Further, the piecewise characteristics of the damper is considered to develop the piecewise linear model, which can reflect the unloading feature of the anti-yaw damper. Finally, a further analysis is made by comparing with the experiment data from the dynamic test bench of an anti-yaw damper.

\section{Establishing the Maxwell Model}

Inside the hydraulic shock absorber, there is the damping valve. When it works, the hydraulic oil flows through the valve orifice forced by the reciprocating piston to generate the damping force. And the rubber joints are mounted at both ends of the hydraulic cylinder.

The performance of the hydraulic shock absorber (damping and stiffness characteristics) can be divided into static and dynamic characteristics. The Shock absorber characteristic curve provided by manufacturers is almost the static one, which ignore stiffness characteristics, so that is no phase change between the damping force and speed. In this case the characteristics of the damper are described as a simple damping model shown as Fig. 1 (a), which can only reflect the low frequency, large amplitude shock absorber characteristics. However, the hydraulic shock absorber has a certain stiffness effect during operation. Because rubber joints stiffness and the oil stiffness supplied by the 
gas bubbles mixed into the oil and the compressed air in the oil storage tank. So there are phase changes between damping fore and vibration speed, performed as the dynamic characteristics.

First of all, the Maxwell model considering both the oil stiffness and the rubber joint stiffness is developed shown as Fig. 1 (c).

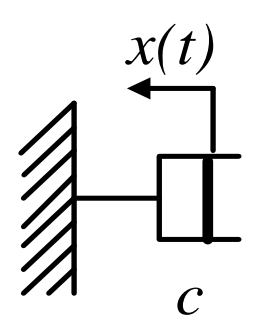

(a)

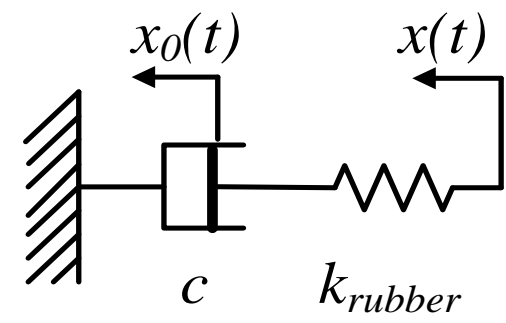

(b)

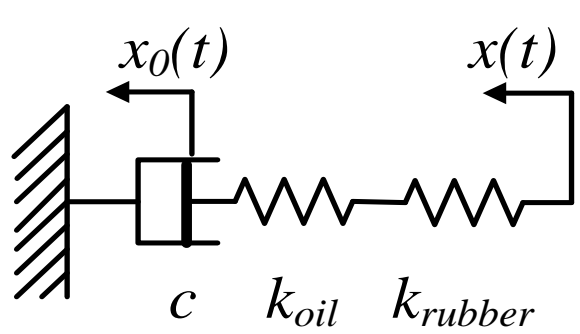

(c)

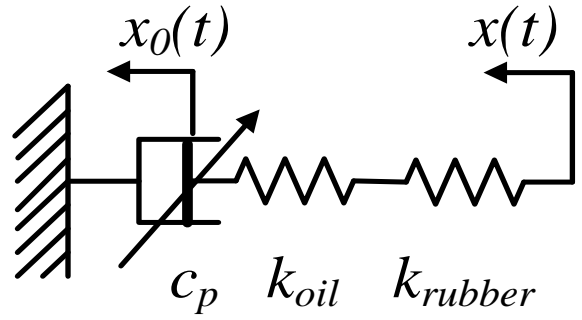

(d)

Fig.1. Computational models of hydraulic shock absorber (a. simple damping model; b. conventional Maxwell model; c. oil-stiffness considered Maxwell model; d. piecewise linear model ).

This model can be described as Eq. 1 .

$m \ddot{x}_{0}=k\left(x-x_{0}\right)-c \dot{x}_{0}$.

Where $m$ is the mass of the piston, $x_{0}$ the displacement of the piston, $x$ the displacement of the end portion, $c$ the linear damper, $k$ the stiffness containing the rubber joint stiffness $\left(k_{\text {rubber }}\right)$ and the oil stiffness $\left(k_{\text {oil }}\right)$ calculated as Eq. 2.

$$
k=\frac{k_{\text {rubber }} k_{\text {oil }}}{k_{\text {rubber }}+k_{\text {oil }}} \text {. }
$$

The inertial force generated by the piston mass can be ignored since it is too small compared with other items. So Eq. 1 can be written as Eq. 3.

$c \dot{x}_{0}+k\left(x_{0}-x\right)=0$.

The displacement of the excitation is assumed as:

$x(\mathrm{t})=A \sin (\omega \mathrm{t})$.

So, the Eq. 5 can be obtained.

$x_{0}=\frac{k^{2} A}{k^{2}+c^{2} \omega^{2}} \sin (\omega t)-\frac{c \omega k A}{k^{2}+c^{2} \omega^{2}} \cos (\omega t)$.

Therefore the shock absorber damping force can be expressed as Eq. 6 .

$$
F=\frac{c \omega k^{2} A}{k^{2}+c^{2} \omega^{2}} \cos (\omega t)+\frac{c^{2} \omega^{2} k A}{k^{2}+c^{2} \omega^{2}} \sin (\omega t) \text {. }
$$

In this case, the damping force $F$ is no longer the same phase with the excitation speed, and there is a phase lag, and the damping force amplitude is reduced accordingly. 


\section{Hysteresis Curve and the Dynamic Characteristics Calculation Method}

To put the displacement of the excitation (Eq. 4) into Eq. 6, the damping force can be described as:

$$
F=\frac{c \omega k^{2} A}{k^{2}+c^{2} \omega^{2}} \sqrt{1-\left(\frac{x}{A}\right)^{2}}+\frac{c^{2} \omega^{2} k}{k^{2}+c^{2} \omega^{2}} x
$$

The hydraulic shock absorber hysteresis curve is a deflected oval shown as Fig. 2, which is calculated by Eq. 7. In this case, the end portion of the damper force $F$ is no longer purely damping force $F_{0}$, the displacement $x$ is also different from the end portion of the piston displacement $x_{0}$. However, the oval area surrounded by $F$ and $x$ still shows the work done by the damping force. The demonstration refers to reference [1].

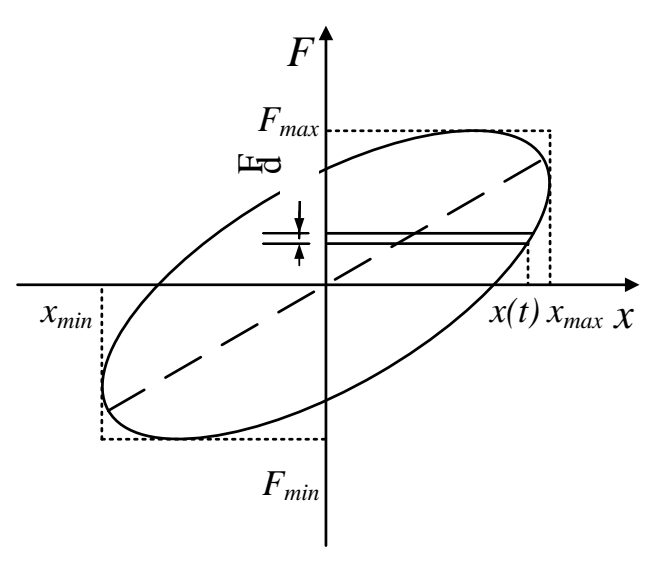

Fig. 2 The hysteresis curve

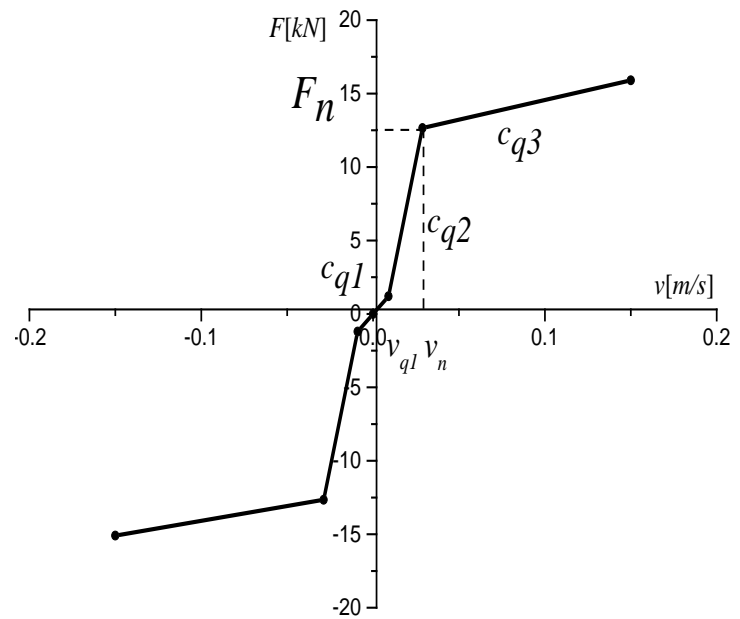

Fig. 3 The anti-yaw damper static damping curve

From the perspective of energy, dynamic damping value $c_{d}$ is shown as Eq. 8 according to the hysteresis curve.

$$
c_{d}=\frac{\int F d x}{\pi \omega A^{2}}
$$

To the dynamic stiffness, the calculation method is shown as Eq. 9, which is linear fitting all the data points, and the slope of the fitted line is the dynamic stiffness of the shock absorber. The benefit of this approach is to avoid the impact of individual data points deviate from the calculation results, especially the test data, and it is possible to accurately reflect the hysteresis curve deflection trend.

$$
k_{d}=\operatorname{ployfit}(x, F)
$$

\section{The Piecewise Linear Model of the Anti-yaw Damper}

Inside the hydraulic shock absorber, especially for the anti-yaw damper, damping valve system is provided with different relief valves, containing corresponding orifice. As the improvement of the piston velocity, the hydraulic pressure within the cylinder increases gradually, and each relief valve open sequentially [5]. Therefore, the damping characteristics generated by the damping valve system show significant piecewise characteristics.

For the anti-yaw damper, mounted longitudinally between the body and the bogie, a smaller damping force is desirable to reduce the rotational torque of bogie, when the vehicle passes a curve line. Whereas, if the damping force increasing excessively with the improving vibration velocity, the risk of derailment will increase correspondingly. So the static damping characteristics of a certain anti-yaw damper are design as Fig. 3. When the vibration speed exceeds a threshold value (unloading speed $v_{n}$ ), the damping force just increases slowly with the vibration velocity, for the reason that the unloading valve of the relief valve opens. 
When the dynamic characteristics of anti-yaw damper is calculated, the piecewise linear characteristics are considered as Fig. 3, and the damping force is expressed as Eq. 10, modified according to Eq. 7. Since in the actual measurement, measurement of movement of the piston is inconvenient, the method commonly used is to use the displacement of the end portion instead of the piston.

$$
F=\left\{\begin{array}{cc}
\frac{c_{q 1} \omega k^{2} A}{k^{2}+c_{q 1}{ }^{2} \omega^{2}} \sqrt{1-\left(\frac{x}{A}\right)^{2}}+\frac{c_{q 1}{ }^{2} \omega^{2} k}{k^{2}+c_{q 1}{ }^{2} \omega^{2}} x & \dot{x} \leq v_{q 1} \\
c_{q 1} v_{q 1}+c_{q 2}\left(\frac{\omega k^{2} A}{k^{2}+c_{q 2}{ }^{2} \omega^{2}} \sqrt{1-\left(\frac{x}{A}\right)^{2}}+\frac{c_{q 2} \omega^{2} k}{k^{2}+c_{q 2}{ }^{2} \omega^{2}} x-v_{q 1}\right) & v_{q 1}<\dot{x} \leq v_{n} \\
c_{q 1} v_{q 1}+c_{q 2}\left(v_{n}-v_{q 1}\right)+c_{q 2}\left(\frac{\omega k^{2} A}{k^{2}+c_{q 3}{ }^{2} \omega^{2}} \sqrt{1-\left(\frac{x}{A}\right)^{2}}+\frac{c_{q 3} \omega^{2} k}{k^{2}+c_{q 3}{ }^{2} \omega^{2}} x-v_{n}\right) & \dot{x}>v_{n}
\end{array}\right.
$$

\section{Comparative Analysis of the Calculation Results and the Test Data}

To verify the accuracy of the piecewise linear model, a dynamic test of one certain anti-yaw damper of the EMUs has been done on the shock absorber dynamic test bench at State Key Laboratory of Traction Power, Southwest Jiaotong University. The damper parameters are shown in Tab. 1.

Tab. 1 Parameters of one certain anti-yaw damper

\begin{tabular}{|c|c|c|}
\hline \multirow{2}{*}{ Piecewise damping $(\mathrm{kNs} / \mathrm{m})$} & $\mathrm{v} \leq 0.006 \mathrm{~m} / \mathrm{s}$ & 213.2 \\
\cline { 2 - 3 } & $0.006<\mathrm{v} \leq 0.02 \mathrm{~m} / \mathrm{s}$ & 440.0 \\
\cline { 2 - 3 } & $\mathrm{v}>0.02 \mathrm{~m} / \mathrm{s}$ (Unloading) & 18.8 \\
\hline Rubber joint stiffness $(\mathrm{MN} / \mathrm{m})$ & \multicolumn{2}{|c|}{35 (two rubber joints in series) } \\
\hline Oil stiffness (MN/m) & \multicolumn{2}{|c|}{37} \\
\hline
\end{tabular}

The end of the damper is fixed on the test bench seat by the rubber joint, and the other end was mounted to the MTS (Mechanical Testing System) hydraulic actuators. The time history of the damping force $F$ and the portion displacement $x$ could be measured and saved in the corresponding calculation collecting module of MTS. Excitation amplitudes of the test were $0.5 \mathrm{~mm}, 2 \mathrm{~mm}$, and Excitation frequencies were $1 \mathrm{~Hz}, 2 \mathrm{~Hz}, 4 \mathrm{~Hz}, 6 \mathrm{~Hz}, 8 \mathrm{~Hz}, 10 \mathrm{~Hz}$. Signal acquisition frequency was $400 \mathrm{~Hz}$, in order to obtain a smooth curve data.

The hysteresis curves of this anti-yaw damper in the vibration amplitude of $0.5 \mathrm{~mm}$ at various frequencies are shown in Fig. 4, and the hysteresis curves in the vibration amplitude of $2 \mathrm{~mm}$ are shown in Fig. 5. In the case of small amplitude, vibration speed is lower than the unloading speed, the damper is not unloading, and the hysteresis curves are approximately oval. However, when the vibration amplitude is large, with frequency increasing, the vibration speed exceeds the unloading speed. In this case, damper uploading, the damping force increases slowly approximated as a horizontal line. 


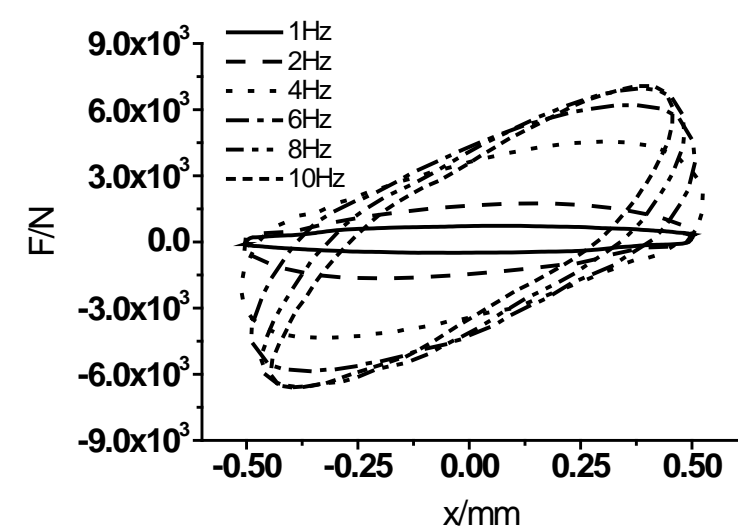

Fig. 4 Test hysteresis curves (not unloading case) For above hysteresis curves, the dynamic damping and the dynamic stiffness can be calculated according to Eq. 8 and Eq. 9. At the same time, the parameters of Tab. 1 are brought into the piecewise linear model, with the same amplitude and frequency as the test, to obtain the results of the model.

The dynamic damping and dynamic stiffness calculated by the piecewise linear model, the conventional Maxwell model, the oil-stiffness considered Maxwell model and the test result are compared in Fig.6- Fig. 9.

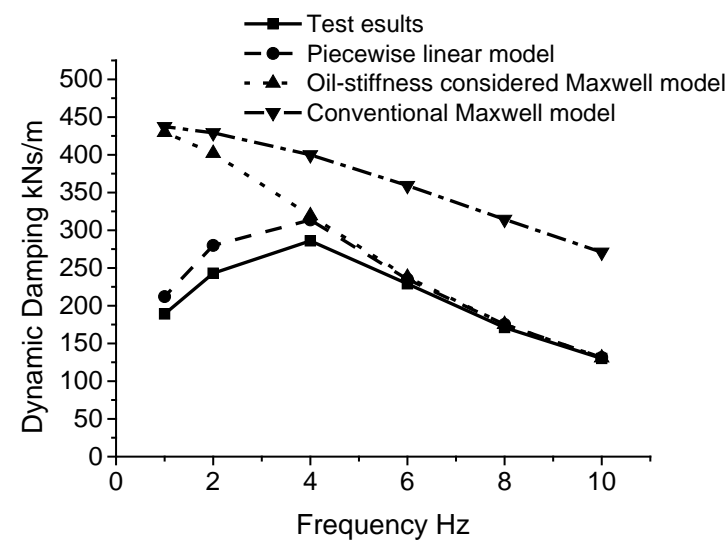

Fig. 6 Dynamic damping curves (not unloading)

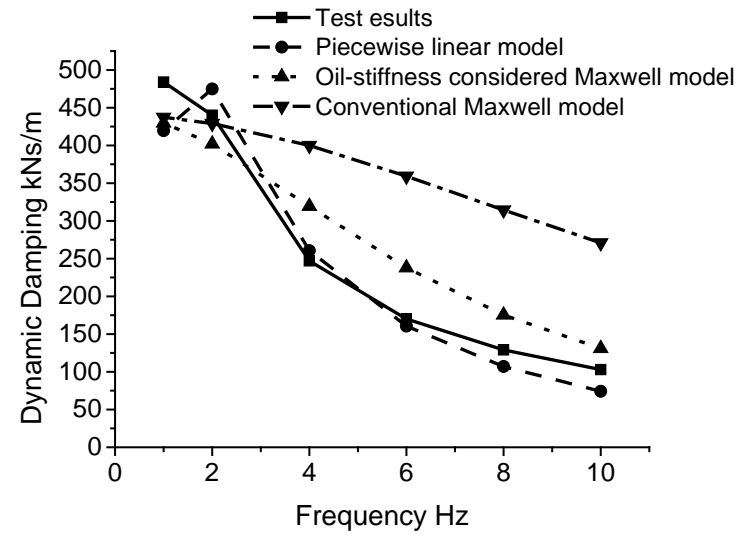

Fig. 8 Dynamic damping curves (unloading)

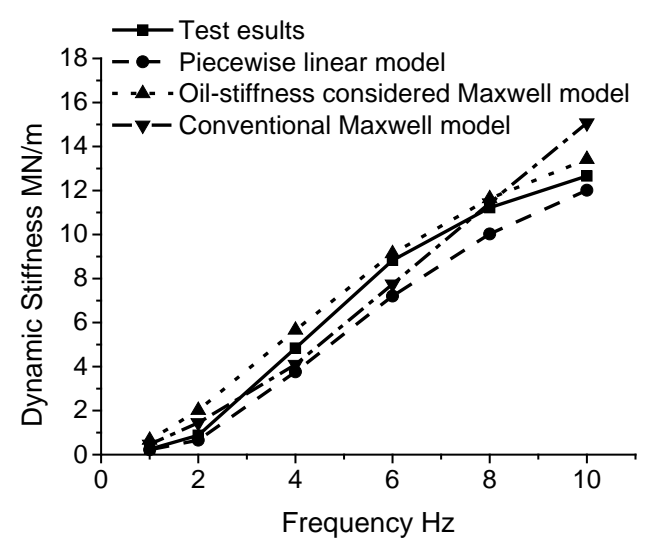

Fig. 7 Dynamic stiffness curves (not unloading)

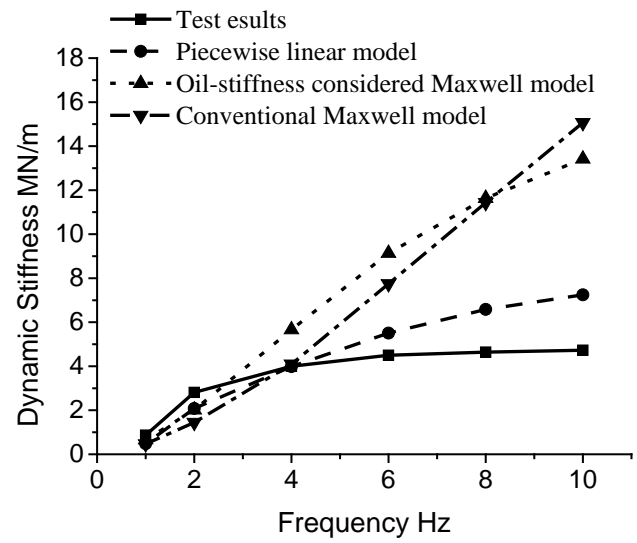

Fig. 9 Dynamic stiffness curves (unloading)

\section{Conclusion}

In this paper, the piecewise linear model was developed by considering the rubber joint stiffness, the oil stiffness and the piecewise characteristics of hydraulic damper. The following conclusion can be achieved by analysis the calculation results and the test results: 
(1) The conventional Maxwell model and the oil-stiffness considered Maxwell model could not reflect the amplitude-dependent dynamic characteristics of anti-yaw damper, and the frequency-dependent dynamic characteristics have large deviation compared with the test.

(2) The piecewise linear model, developed in this paper, can accurately reflect both the amplitude-dependent and frequency-dependent dynamic characteristics, which is in good agreement with the test results.

(3) The piecewise linear model can also accurately reflect the piecewise characteristics of hydraulic damper, e.g. unloading case, caused by the sequentially opening of the relief valves inside the anti-yaw damper.

\section{Acknowledgements}

This work has been supported by the State Key Program of National Natural Science of China (61134002), the National Key Basic Research Program of China (973 Program) (2011CB711100) and Innovation Group of Ministry of Education funded project (IRT1178).

\section{References}

[1] G.Z. Yang, F.T. Wang. Hydraulic Dampers for Railway Rolling Stock [M]. China Railway Publishing House, Beijing (2003)

[2] Alonso. Yaw Damper Modelling And its Influence on the Railway Dynamic Stability [J]. Vehicle System Dynamics, 49(9), 1367-1387, 2011.

[3] C.H. Huang. Study on Vibration Reduction Technologies for High Speed Cars [D]. Chengdu: Southwest Jiaotong University,2012.

[4] G.D. Lu. Effect of Series Stiffness on Features of Hydraulic Dampers [J]. Rolling Stock, $2007.45(2)$.

[5] G.X. Xu. Parametric Modeling of High-speed Train Hydraulic Dampers [D]. Nanchang: Nanchang University, 2010.

[6] D.C. Liu. Experimental Study on Mechanical Properties of Springs for Railway Vehicles[D]. Chengdu: Southwest Jiaotong University, 2014. 